\title{
Cold Atmospheric Plasma Prevents Wrinkle Formation via an Antiaging Process
}

\author{
Sang Gyu Hwang, ${ }^{\text {a }}$ Jung Ho Kim, ${ }^{\mathrm{b}}$ Soo Youn Jo, ${ }^{\text {a }}$ Young Jae Kim, ${ }^{\text {a,* }}$ \& \\ Chong Hyun Won ${ }^{a, *}$ \\ aDepartment of Dermatology, Asan Medical Center, University of Ulsan College of Medicine, \\ Seoul 05505, Korea; 'Department of Teaching and Learning, School of Culture, Education, and \\ Human Development, New York University, New York, NY 10003 \\ *Address all correspondence to: Chong Hyun Won, MD, PhD, Department of Dermatology, Asan Medical \\ Center, University of Ulsan College of Medicine, 88, Olympic-ro 43-gil, Songpa-gu, Seoul 05505, Korea; \\ Tel.: +82-2-3010-3460; Fax: +82-2-486-7831, E-mail: drwon@amc.seoul.kr; or Young Jae Kim, MD, Department of \\ Dermatology, Asan Medical Center, University of Ulsan College of Medicine, 88, Olympic-ro 43-gil, Songpa-gu, Seoul \\ 05505, Korea; Tel.: +82-2-3010-0269; Fax: +82-2-486-7831, E-mail: assayoungjae@naver.com
}

\begin{abstract}
Cold atmospheric plasma is a material that generates free radicals through the ionization of air. Despite the application of this material in various medical fields, the precise mechanism underlying its effect at the cellular level is not fully understood. To date, it is known to stimulate the expression of antioxidant transcription factors such as nuclear factor erythroid 2-related factor 2 (Nrf2), forkhead box class O 3a (FOXO3a), and heme oxygenase 1 (HO-1). In this study, we aimed to assess whether and how cold atmospheric plasma affects damaged cells, particularly after exposure to UV radiation. We found that it can promote mitochondrial health by controlling the stability of the membrane potential of mitochondria against UV radiation. Moreover, cold atmospheric plasma prevents senescence in fibroblasts exposed to UV radiation through the upregulation of $\mathrm{p} 53$. It also exhibits hormetic effects (i.e., beneficial effects at low doses) on MMP and collagen in senescent fibroblasts exposed to UV radiation. In conclusion, cold atmospheric plasma treatment not only protects fibroblasts from free radicals but also represses the aging of these cells in a UV-radiation-induced aging process.
\end{abstract}

KEY WORDS: cold atmospheric plasma, wrinkle, antiaging, collagen, MMP, skin

\section{INTRODUCTION}

The skin is the outermost organ of the human body and acts as a first defense during exposure to sunlight or UV radiation. One of the most notable consequences of skin aging is wrinkle formation, which is closely related to cosmetic concerns. Specifically, fibroblasts exposed to ultraviolet (UV) radiation exhibit overexpression of matrix metallopeptidases (MMPs) and disruption of the homeostasis between collagen and MMP. ${ }^{1}$ Although excessive generation of radicals, such as reactive oxygen species (ROS), is harmful, an adequate level of ROS production sometimes triggers the activation of signaling pathways related to antioxidation and the antiaging process. Nuclear factor erythroid 2-related factor 2 (Nrf2) is a representative transcription factor that contributes to antioxidation, whereas the forkhead box class O (FOXO) transcription factor contributes to cellular resistance to stress, DNA damage repair, cell death, and apoptosis. 
Mitochondria are powerhouses within cells that produce adenosine triphosphate (ATP). The depletion of ATP through external stimulation, such as exercise or radical stress, leads to the activation of the adenosine monophosphate (AMP-) -activated protein kinase (AMPK) pathway, which is a sensor of intracellular adenosine nucleotide levels. This pathway plays a role in the redox system and promotes FOXO activation. ${ }^{2}$ Damaged or dysfunctional mitochondria induce aging, cancer, metabolic diseases, and neurodegenerative diseases. ${ }^{3}$ The antioxidant function of mitochondria occurs through the peroxisome proliferator-activated receptor gamma coactivator 1-alpha (PGC-1 $\alpha$ ) pathway and can suppress senescence. ${ }^{4}$ However, excessive radical species can lead to dysfunction of the mitochondrial electron transport chain (ETC) and respiration dysfunction, ${ }^{5}$ and eventually to the reduction of mitochondrial membrane potential. ${ }^{6}$ In addition, senescent cells contain a high proportion of damaged mitochondria, which results in aging. Also, the accumulation of senescent cells in tissues may lead to carcinogenesis via the activation of oncogenes. To prevent this, the tumor suppressor protein p53 inhibits tumor development and regulates repair and apoptosis in cells based on the level of damage.

We investigated the effect of cold atmospheric plasma in fibroblasts exposed to UV radiation and demonstrated its effectiveness as an antiaging material for the skin.

\section{MATERIALS AND METHODS}

\section{A. Primary Cell Culture}

Human dermal fibroblast primary cells were extracted from adult skin tissue, mainly from the back area (unexposed to solar UV radiation), of 30-37-year-old subjects. Subsequently, the epidermis was removed and the dermis was washed in serially diluted ethanol. The dermis was incubated in a T75 mammalian cell culture plate at $37^{\circ} \mathrm{C}$ in a $5 \% \mathrm{CO}_{2}$ incubator. Primary fibroblasts were incubated in DMEM containing $10 \% \mathrm{FBS}$ and $1 \%$ antibiotics, and were cultured at $37^{\circ} \mathrm{C}$ in a $5 \% \mathrm{CO}_{2}$ incubator. Fibroblasts between passages 3 and 13 were used in this study.

\section{B. Cold Atmospheric Plasma Device}

The cold atmospheric plasma (CAP) medical device used here, manufactured by MediPL (model, ESM050), consisted of a multi plasma jet that was generated by four feeding points. The device uses argon gas and a resonant frequency of $2.45 \mathrm{GHz}$, and is operated in three modes. The plasma power used is $2 \mathrm{~W}$ in mode $1,6 \mathrm{~W}$ in mode 2 , and $10 \mathrm{~W}$ in mode 3 . The argon gas flow is the same across the three modes $(1 \mathrm{~L} / \mathrm{min})$.

\section{UV Exposure and Plasma Treatment}

Fibroblasts were seeded in 30-mm dishes and grown to a confluence of $70 \%-80 \%$. The cell medium was treated with plasma for $0.25,0.5,1$, and 2 min at an argon gas flow of $0.5 \mathrm{slm}$. With the exception of normal fibroblasts, confluent fibroblasts were exposed to 
UV radiation for $1 \mathrm{~min} ; 4 \mathrm{~h}$ later, the cell culture medium was treated with plasma and transferred into the UV-radiation-exposed fibroblast culture. Four TL20W/12RS UV lamps (Philips) and a UV meter (YK-34UV, Lutron Electronics) were used. The UV radiation energy was calculated as $1.4 \mathrm{~mW} \times 60 \mathrm{~s}=84 \mathrm{~mJ} / \mathrm{cm}^{2}$.

\section{RT-PCR}

The cells were collected from the 30 -mm dish containing human dermal primary fibroblasts. mRNA was extracted using a Trizol solution. cDNA was synthesized using a mixture of RT-GO, oligo dTs, DTT, and sterilized water. RT-PCR was performed using cDNA, forward and backward primers, dNTPs, Ex Taq polymerase, 10× Ex Taq buffer, and DW. The intensity of bands was quantified using ImageJ software. (https://imagej.nih.gov/ij/ download.html). The sequences of the primers used in this study are described in Table 1.

\section{E. Western Blot Analysis}

Western blot was performed using whole-cell protein extracts and cytosol/nuclear proteins from primary fibroblasts cultured in $30-\mathrm{mm}$ dishes. The primary antibodies used here were anti-Sirt1, anti-FOXO3a, anti-phosphorylated-Akt (ser473), anti-p53, antiiNOS, anti-Bcl2, anti-caspase3 (Cell Signaling), anti-PGC-1a (Bio Vision), anti-phospho-AMPKa1/2 thr172 (Santa Cruz Biotechnology), anti-Nrf2, and anti-TNF-alpha (Abcam) polyclonal antibodies, as well as a monoclonal anti- $\beta$-actin antibody (SigmaAldrich). Band intensity was quantified using ImageJ software.

\section{F. Nuclear/Cytosolic Fractionation}

Nuclear and cytosolic fractions of dermal fibroblasts were prepared using a nuclear/ cytosolic fractionation kit (Cell Biolabs).

\section{Cytoplasmic Fraction}

Fibroblasts were collected by centrifugation for $5 \mathrm{~min}$ and washed with PBS. After removal of the supernatant, the cell pellet was gently resuspended in 500- $\mu 11 \times$ cytosol extraction buffer. The suspension was transferred into a micro-centrifuge tube and incubated on ice for $10 \mathrm{~min}$. Cell lysis reagent $(25 \mu \mathrm{l})$ was added, and cells were vortexed for $10 \mathrm{~s}$ and centrifuged for $10 \mathrm{~min}$ at $800 \times g$. The supernatant was then transferred carefully into a clean micro-centrifuge tube.

\section{Nuclear Fraction}

Cell lysis buffer $(25 \mu \mathrm{l})$ was added to the remaining pellet, and the cells were vortexed for $10 \mathrm{~s}$ and then centrifuged for $10 \mathrm{~min}$ at $800 \times g$. The supernatant was aspirated and discarded. The nuclear pellet was resuspended in 100- $\mu 11 \times$ nuclear extraction buffer, 
Hwang et al.




kept on ice for $30 \mathrm{~min}$, and vortexed for $10 \mathrm{~s}$ at 10-min intervals. This was followed by centrifugation for $30 \mathrm{~min}$ at $14,000 \times g$ and careful transfer of the supernatant to a clean micro-centrifuge tube.

\section{G. Immunocytochemistry}

Fibroblasts were fixed in 4\% formalin for $1 \mathrm{~h}$ and permeabilized in $0.1 \%$ Triton $\mathrm{X}-100$ for $15 \mathrm{~min}$. Cells were incubated with the primary antibody for $1 \mathrm{~h}$, with the secondary antibody for $45 \mathrm{~min}$, and with $1 \times$ DAPI staining for $30 \mathrm{~min}$. Subsequently, mounting solution was added and the microscope slide was covered with a cover slip. Slides were observed using an Olympus IX81 fluorescence microscope.

\section{H. ELISA Measurements}

Human MMP1 and MMP3 ELISA kits were purchased from Abcam. The cultured cell supernatants of normal, UV-irradiated, and UV/plasma-treated fibroblasts were used for ELISA of MMP1 and MMP3 according to the manufacturer's protocol.

\section{Cellular Senescence Assay}

The cellular senescence (SA-beta-gal staining) detection kit was purchased from Cell Biolabs. Human dermal fibroblasts were seeded in $30-\mathrm{mm}$ dishes and incubated at $37^{\circ} \mathrm{C}$ in a $5 \% \mathrm{CO} 2$ incubator until approximately $60 \%-70 \%$ confluence. As per the protocol recommended by the manufacturer, a $1 \times$ fixing solution (diluted from the $100 \times$ stock) and a cell staining working solution (made up of four staining solutions $[\mathrm{A}, \mathrm{B}, \mathrm{C}$, and D], X-gal solution, and $\mathrm{H}_{2} \mathrm{O}$ ) were prepared. Dermal fibroblasts were washed with $1 \times$ PBS and fixed in the $1 \times$ fixing solution. After incubation, the fixing solution was removed and the fibroblasts were washed with $1 \times$ PBS, covered with 2-ml cell staining working solution, and incubated at $37^{\circ} \mathrm{C}$. After removal of the working solution, the cells were washed with $1 \times$ PBS and the senescent fibroblasts were observed using light microscopy.

\section{J. JC-1 Dye Staining}

A JC-1 assay kit (Cell Biolabs) was used for measurement of the mitochondrial membrane potential. Human dermal fibroblasts were seeded in $30-\mathrm{mm}$ dishes and incubated at $37^{\circ} \mathrm{C}$ in a $5 \% \mathrm{CO} 2$ incubator until approximately $60 \%-70 \%$ confluence. As per the protocol recommended by the manufacturer, the medium of the fibroblast cultures was aspirated into a $60-\mathrm{mm}$ culture dish and the cells were washed three times with $1 \times$ PBS and incubated in 2-ml $1 \times$ fixing solution at room temperature for $5 \mathrm{~min}$. After removal of the fixing solution, the cells were washed three times and 2-ml cell staining working solution was added followed by incubation at $37^{\circ} \mathrm{C}$ in the dark for 4 $\mathrm{h}$ or overnight. Finally, the cell staining working solution was removed, the cells were

Volume 10, Issue 2, 2020 
washed three times with $1 \times$ PBS, and the senescent fibroblasts were observed using light microscopy.

\section{K. Measurement of RNS and Nitrous Oxide Generation}

Reactive nitrogen species (RNS) and nitrous oxide (NO) assay kits, purchased from Cell Biolabs, were used to measure RNS and NO according to the manufacturer's instructions.

\section{Standard Deviation Analysis}

Standard deviation analysis was performed using Microsoft Excel. Significance was set at $P<0.05$, as calculated using Excel.

\section{RESULTS}

\section{A. Type 3 Collagen Increase}

Wrinkles are a phenotype of severe degradation of type 1 collagen in fibroblasts with long-term exposure to UV radiation. We observed changes in both type 1 and type 3 collagen levels and then determined at what CAP treatment time they occurred. As shown in Figs. 1A and 1B, all fibroblasts, with the exception of normal dermal fibroblasts, were exposed to UV radiation for $1 \mathrm{~min}$; after $4 \mathrm{~h}$, some were treated with the CAP-treated medium for various periods $(15,30,60$, and $120 \mathrm{~s})$. An RT-PCR analysis performed 48 $\mathrm{h}$ later showed that the highest amount of collagen 1 synthesis occurred in fibroblasts treated with CAP for 1 min.

Both type 1 and type 3 collagen levels were increased at $48 \mathrm{~h}$ with the change to the CAP-treated medium $4 \mathrm{~h}$ after exposure to UV irradiation for $1 \mathrm{~min}$ (Fig. 1C). Type 3 collagen is formed earlier than type 1 collagen and is abundant in young skin. Accordingly, we observed that the level of type 3 collagen was higher at $24 \mathrm{~h}$ vs. $48 \mathrm{~h}$ of culture, as assessed by RT-PCR and fluorescence microscopy (Figs. 1D and 1E). Because collagens are produced in dermal fibroblasts, are secreted outside cells, and participate in formation of the extracellular matrix (ECM), we stained type 3 collagen in the absence of the Triton X-100 surfactant. At $24 \mathrm{~h}$ of culture, the collagen was increased to a greater extent in the UV/plasma-treated fibroblasts than in the fibroblasts exposed to UV irradiation alone. However, the type 1 collagen level was changed significantly at this time point (Fig. 1F). Moreover, through plasma treatment, MMP1 and MMP3 were downregulated at $24 \mathrm{~h}$ of culture, with the downregulation of MMP3 showing statistical significance as assessed by ELISA. MMP1 decreased by $13.2 \%$ and MMP3 decreased by $35.8 \%(* P=0.03<0.05$; Fig. $1 \mathrm{G})$.

Taken together, the results reveal that levels of the collagen proteins definitely increased in UV/plasma-treated fibroblasts whereas both MMP1 and MMP3 levels decreased. Therefore, upregulation of type 1 and type 3 collagen and downregulation of 
(A)

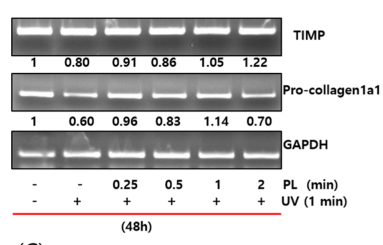

(C)

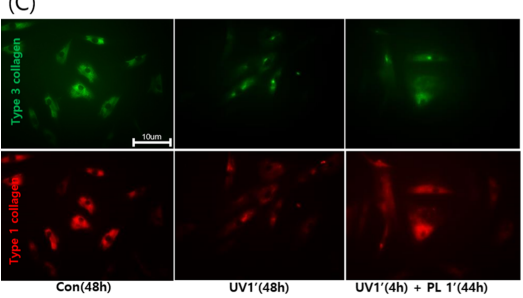

(D)

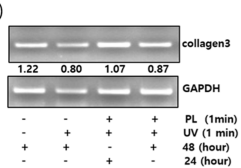

(E)



(F)
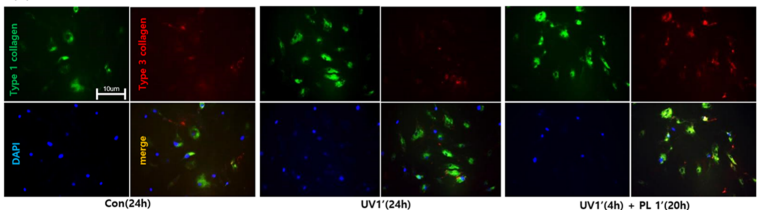

(G)

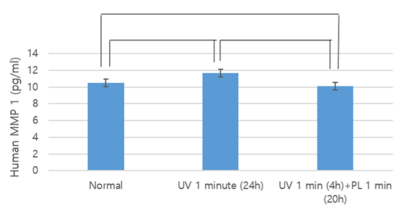

(B)

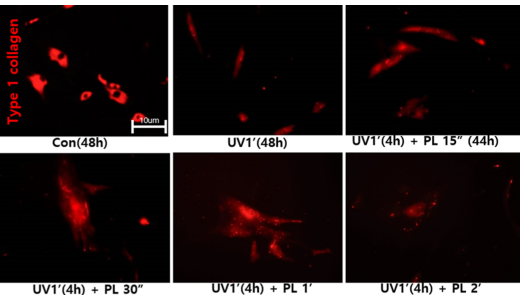

(1)(4h) + PL 30

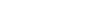$$
\text { 列 }
$$ \\ .}


MMP1 and MMP3 suggest that CAP is effective in preventing the formation of wrinkles by UV-exposed photo-aged fibroblasts.

\section{B. Decrease in ROS And NO and Increase in Type 3 Collagen}

We focused on the production of type 3 collagen because this protein is not abundant in adult skin. Because ROS induce aging, collagen degradation, and production of NO (which is also a free radical), we examined the generation of ROS and NO after exposure to UV radiation. We also investigated changes in ROS and NO caused by treatment with CAP administered at $24 \mathrm{~h}$ after UV exposure. Both NO and ROS increased in UVirradiated fibroblasts; however, this effect was attenuated by the plasma treatment (Figs. $2 \mathrm{~A}$ and $2 \mathrm{~B}$ ). We found that the plasma contributed to the reduction of the oxidative and nitrosative stress caused by UV radiation. Moreover, fluorescence microscopy and western blot showed that the nuclear translocation of Nrf2 was more pronounced in UV/ plasma-treated fibroblasts than in UV-irradiated fibroblasts. Type 3 collagen production was also highest in UV/plasma-treated fibroblasts. In contrast, it was barely detectable in UV-irradiated fibroblasts (Figs. 2C and 2D).

Because heme oxygenase 1 (HO-1) is an antioxidant product of the transcriptional activity of $\mathrm{Nrf} 2$, we assessed the expression of this protein in our system. The expression level of HO-1 decreased in the UV-irradiated fibroblasts compared with the normal fibroblasts. However, the UV/plasma-treated fibroblasts revealed a relatively increased level of HO-1 expression compared with UV-irradiated fibroblasts (Fig. 2E).

Subsequently, we examined whether the plasma treatment had an antiaging effect. Sirt1 (a mammalian NAD ${ }^{+}$-dependent histone deacetylase) is a master protein that regulates many target proteins, with antioxidative and antiaging effects. Therefore, we expected that, in addition to Nrf2 and HO-1, Sirt1 would assert is antiaging effect once activated by the plasma treatment. We found that Sirt1 expression was increased in UV/plasma-treated fibroblasts; however, we detected a low Sirtl level in UV-irradiated fibroblasts (Fig. 2F). We also confirmed that the FOXO3a protein was upregulated not only in the normal fibroblasts but also in the UV/plasma-treated fibroblasts, as assessed using Western blotting (Fig. 2F).

Together with the results reported above, these findings suggest that CAP has the capacity to protect fibroblasts from UV-induced oxidative stress. Moreover, our results strongly suggest that plasma is effective in promoting the production of type 3 collagen via the expression of Sirt1 and Nrf2.

\section{Cold Atmospheric Plasma's Contribution to the Maintenance of Healthy Mitochondria}

The mitochondrial membrane potential is strongly associated with the apoptosis caused by aging. Based on the JC-1 dye assay, we showed that UV-irradiated fibroblasts have a low membrane potential whereas UV/plasma-treated fibroblasts have a high membrane potential. (Fig. 3A). Moreover, caspase3 was upregulated to a greater extent in 
(A)

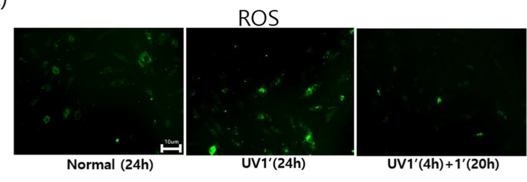

(B)

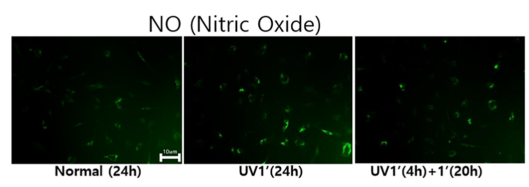

(C)
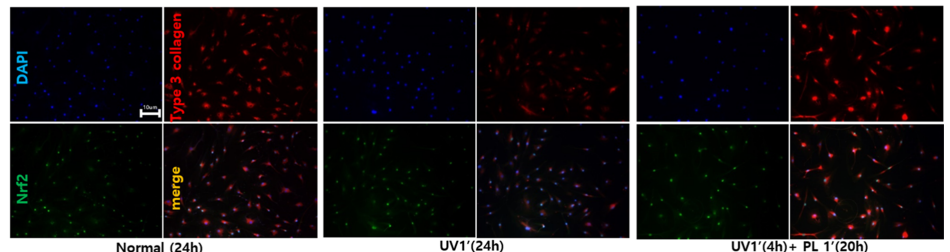

(D)
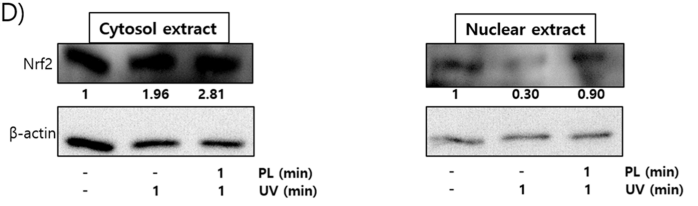

(E)
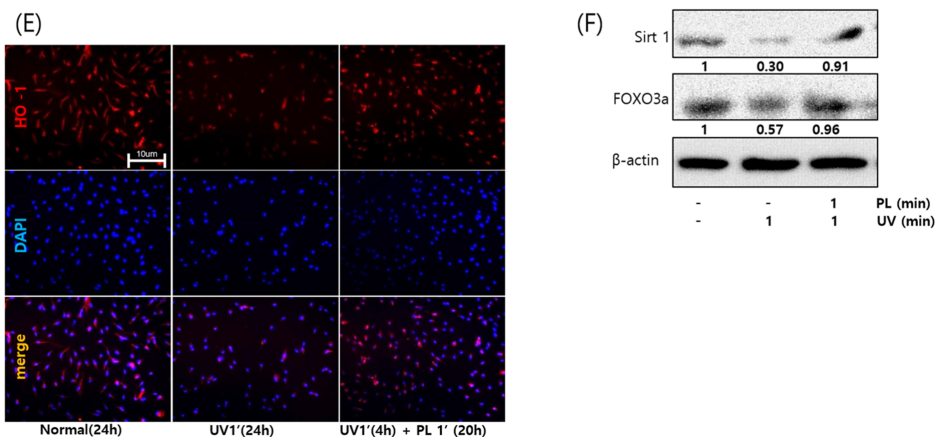

FIG. 2: The decrease in levels of ROS and NO after CAP treatment contributed to the production of type 3 collagen. (A) UV radiation induced excessive ROS production, whereas the plasma medical device decreased ROS generation (Olympus IX71, 10×, 0.30, 1.6×). (B) NO generation followed a pattern similar to that of ROS generation (Olympus IX71, 10×, 0.40, 1.6×). Nrf2 was translocated to the nucleus of UV/plasma-treated fibroblasts, leading to production of type 3 collagen. (C) Fluorescence image (Olympus IX71, 10×, 0.40, 1.6×). (D) Nrf2 expression in the cytosol and nucleus as assessed by western blotting. (E) HO-1 expression is elevated in normal and UV/plasma-treated fibroblasts but very low in UV-irradiated fibroblasts (Olympus IX71, $20 \times, 0.45$ ). (F) Sirt1 and FOXO3a were upregulated to a greater extent in UV/plasma-treated fibroblasts versus UV-irradiated fibroblasts.

Volume 10, Issue 2, 2020 
(A)

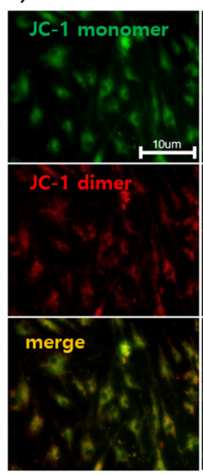

Normal (24h)

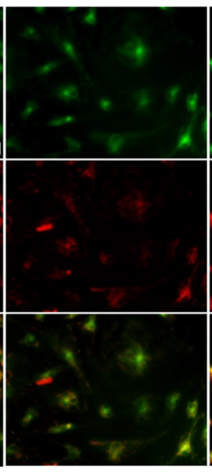

UV1'(24h)

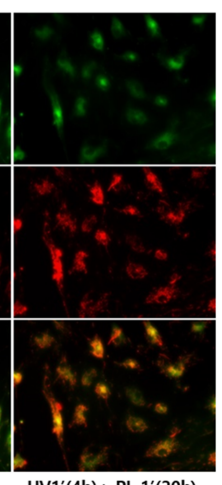

UV1'(4h)+ PL 1'(20h)
(B)

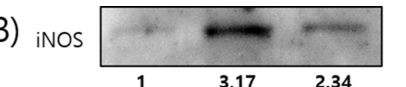

caspase 3

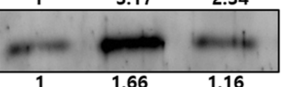

$\mathrm{Bcl} 2$



$\beta$-actin

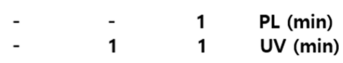

FIG. 3: CAP inhibited disruption of the mitochondrial membrane potential. (A) The mitochondrial membrane potential was high in UV-irradiated fibroblasts treated subsequently with the plasma medical device (Olympus IX71, 20×, 0.45, 1.6×). (B) Apoptosis-related proteins within mitochondria exhibited the highest expression levels in UV-irradiated fibroblasts.

UV-irradiated fibroblasts than in UV/plasma-treated fibroblasts. The antiapoptotic protein $\mathrm{Bcl} 2$ was also upregulated to a greater extent in UV-irradiated versus UV/plasmatreated fibroblasts (Fig. 3B). Using western blotting, we found that expression of the iNOS protein followed a pattern similar to that of NO generation (Fig. 3B). These results show that plasma treatment contributes to the prevention of mitochondrial damage and to the maintenance of mitochondrial function against UV radiation.

\section{Senescence of Fibroblasts Exposed to UV Radiation}

We observed that senescence increases in fibroblasts exposed to UV radiation and decreases in fibroblasts treated with plasma, as assessed via SA- $\beta$-gal (beta-galactosidase) assay (Fig. 4A). Here, p53 was upregulated to a greater extent in UV/plasmatreated compared with UV-irradiated fibroblasts (Fig. 4B). Because p53 is activated as

(A)
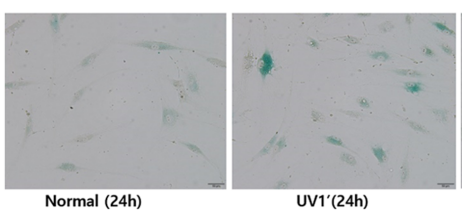

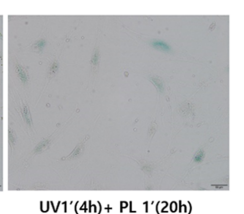

(B)

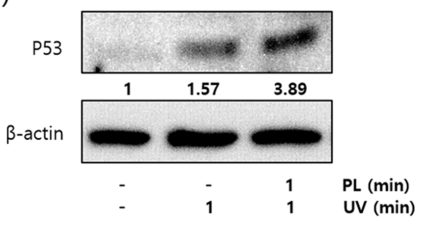

FIG. 4: CAP inhibited senescence in UV-irradiated fibroblasts. (A) Senescence was decreased by the plasma medical device in both high-passage and low-passage fibroblasts (Olympus BX53, $10 \times, 0.3$ ). (B) Expression of the tumor suppressor protein $\mathrm{p} 53$ was increased by the plasma medical device in UV-irradiated fibroblasts. 
a transcription factor for the double functions of apoptosis and DNA repair, we propose that CAP has similar functions. Therefore, we suggest that, through UV exposure, CAP contributes to the prevention of senescence.

\section{DISCUSSION}

The main pathogenesis of wrinkles is based on collagen breakdown by MMPs and/ or the inhibition of de novo collagen production. Notably, exposure to UV radiation causes overexpression of ROS, which contributes to further wrinkle formation during the photo-aging process. Although we saw no change in collagen RNA levels, there was a significant change in protein levels. We suggest that this is because UV disturbs protein production. MMP1 and MMP3 are the main enzymes activated during the photoaging process. In particular, MMP1 breaks down type 1 and type 3 collagen. ${ }^{7,8}$ We found that fibroblasts exposed to UV radiation and treated with CAP exhibit a decrease in both ROS production and MMP expression (Figs. $1 \mathrm{G}$ and 2A), which implies that the downregulation of MMP by CAP may cause less collagen breakdown, thus preventing further wrinkle formation.

In addition, we observed in fibroblasts upregulation of both Sirt1 and Nrf2, antioxidant transcription factors, resulting in a reduction of ROS. It is reported that Sirt1 enhances the production of antioxidants mediated by FOXO. ${ }^{9}$ Moreover, its inhibition increases the production of both ROS and NO, ${ }^{10}$ suggesting that its upregulation causes a decrease in the ROS produced in UV-irradiated fibroblasts. As indicated in our study, despite exposure to UV radiation, CAP-treated fibroblasts show increased expression of Sirt1 and HO-1 (Fig. 2E and 2F). Therefore, the upregulation of FOXO3a, Nrf2, and Sirt1 by CAP treatment seems to play a significant role in the transcription of antioxidative genes.

As shown in Fig. 3, dysfunctional mitochondria in UV-irradiated fibroblasts increase the expression of caspase 3 and $\mathrm{Bcl} 2$. Although we could not confirm direct apoptosis in fibroblasts exposed to UV radiation, the accumulation of caspase 3 and $\mathrm{Bcl} 2$ may provoke cellular senescence. It is known that mitochondria are important players in the senescence of UV-irradiated fibroblasts, ${ }^{11}$ and that dysfunctional mitochondria steadily accumulate with the advancement of senescence. ${ }^{12}$ Based on these findings, we propose that plasma treatment results in the downregulation of $\mathrm{Bcl} 2$ and caspase 3 , in turn inhibiting senescence and carcinogenesis.

In general, tumor cells exhibit mutational defects in the $p 53$ gene. UV-irradiated cells undergo DNA damage, which causes cellular senescence ${ }^{13}$ and carcinogenesis. ${ }^{14}$ As shown in Fig. 4, CAP treatment increases the expression of p53; therefore, it is able to inhibit the senescence process in UV-irradiated fibroblasts.

In conclusion, CAP enhances the preservation of collagen and the decrease in MMPs in skin fibroblasts exposed to UV radiation. It may also restore the functioning of UV-damaged mitochondria and repair DNA damage to the $p 53$ gene. These processes may minimize further wrinkle formation and contribute to the prevention of cellular senescence in photo-aged skin.

Volume 10, Issue 2, 2020 


\section{ACKNOWLEDGMENTS}

This study was supported by a grant (Grant Nos. 2016-704, 2017-704) from the Asan Institute for Life Sciences, Asan Medical Center, Seoul, Korea. The study was sponsored by the Korean Ministry of Science and ICT as part of the project Development of Novel Medical Devices Using Low Temperature Microwave Plasma Technology (NRF2015M3A9E2066978). In addition, it was supported by a grant (Grant No. 2019IF0586) from the Asan Institute for Life Sciences and Corporate Relations of the Asan Medical Center, Seoul, Korea.

\section{REFERENCES}

1. Philips N, Auler S, Hugo R, Gonzalez S. Beneficial regulation of matrix metalloproteinases for skin health. Enzyme Res. 2011;2011:427285.

2. Zhao Y, Hu X, Liu Y, Dong S, Wen Z, He W, Zhang S, Huang Q, Shi M. ROS signaling under metabolic stress: Cross-talk between AMPK and AKT pathway. Mol Cancer. 2017;16(1):79.

3. Kudryavtseva AV, Krasnov GS, Dmitriev AA, Alekseev BY, Kardymon OL, Sadritdinova AF, Fedorova MS, Pokrovsky AV, Melnikova NA, Kaprin AD, Moskalev AA, Snezhkina AV. Mitochondrial dysfunction and oxidative stress in aging and cancer. Oncotarget. 2016;7(29):44879-905.

4. Rabinovitch RC, Samborska B, Faubert B, Ma EH, Gravel SP, Andrzejewski S, Raissi TC, Pause A, St-Pierre J, Jones RG. AMPK maintains cellular metabolic homeostasis through regulation of mitochondrial reactive oxygen species. Cell Rep. 2017;21(1):1-9.

5. Ghasemi M, Mayasi Y, Hannoun A, Eslami SM, Carandang R. Nitric oxide and mitochondrial function in neurological diseases. Neuroscience. 2018;376:48-71.

6. Takabayashi A, Kawai Y, Iwata S, Kanai M, Denno R, Kawada K, Obama K, Taki Y. Nitric oxide induces a decrease in the mitochondrial membrane potential of peripheral blood lymphocytes, especially in natural killer cells. Antioxid Redox Signal. 2000;2(4):673-80.

7. Elkington PT, Ugarte-Gil CA, Friedland JS. Matrix metalloproteinases in tuberculosis. Eur Respir J. 2011;38(2):456-64.

8. Pittayapruek P, Meephansan J, Prapapan O, Komine M, Ohtsuki M. Role of matrix metalloproteinases in photoaging and photocarcinogenesis. Int J Mol Sci. 2016;17(6):868.

9. Salminen A, Kaarniranta K, Kauppinen A. Crosstalk between oxidative stress and SIRT1: Impact on the aging process. Int J Mol Sci. 2013;14(2):3834-59.

10. Becatti M, Barygina V, Mannucci A, Emmi G, Prisco D, Lotti T, Fiorillo C, Taddei N. Sirt1 protects against oxidative stress-induced apoptosis in fibroblasts from psoriatic patients: A new insight into the pathogenetic mechanisms of psoriasis. Int J Mol Sci. 2018;19(6):1572.

11. Correia-Melo C, Marques FD, Anderson R, Hewitt G, Hewitt R, Cole J, Carroll BM, Miwa S, Birch J, Merz A, Rushton MD, Charles M, Jurk D, Tait SWG, Czapiewski R, Greaves L, Nelson G, Bohlooly-YM, Cuenca SR, Puig AV, Mann D, Saretzki G, Quarato G, Green DR, Adams PD, von Zglinicki T, Korolchuk VI, Passos JF. Mitochondria are required for pro-ageing features of the senescent phenotype. EMBO J. 2016;35(7):724-42.

12. Korolchuk VI, Miwa S, Carroll B, von Zglinicki T. Mitochondria in cell senescence: Is mitophagy the weakest link? EBioMedicine. 2017;21:7-13.

13. Lei D, Huang Y, Xie H, Yi Y, Long J, Lin S, Huang C, Jian D, Li J. Fluorofenidone inhibits UV-A induced senescence in human dermal fibroblasts via the mammalian target of rapamycin-dependent SIRT1 pathway. J Dermatol. 2018;45(7):791-8.

14. Seviour EG, Lin SY. The DNA damage response: Balancing the scale between cancer and ageing. Aging. 2010;2(12):900-7. 